\title{
Secondary variants - in defense of a more fitting term in the incidental findings debate
}

\begin{abstract}
Gabrielle M Christenhusz ${ }^{\star, 1}$, Koenraad Devriendt ${ }^{2}$ and Kris Dierickx ${ }^{1}$
New genetic technologies are capable of returning far more information than the single answer to the single question posed when conducting a given genetic test. Genetics contexts consequently stand on the brink of an explosion of what have traditionally been called 'incidental findings'. However, the continued use of this term is controversial. Various replacements for 'incidental findings' have been attempted, but none with widespread success. Agreement on terminology and definitions is vital so that the legal and ethical debate around incidental findings can proceed. We highlight the difficulties raised by the various terms currently used as alternatives, and end by defending our choice for the term 'secondary variants'.
\end{abstract}

European Journal of Human Genetics (2013) 21, 1331-1334; doi:10.1038/ ejhg.2013.89; published online 22 May 2013

Keywords: incidental findings; secondary variants; genetics

\section{THE IMPORTANCE OF FINDING THE RIGHT NAME}

S econdary variants. Unexpected or offStarget results. Unanticipated or unsought for or unrelated findings. Abnormalities existing, potential, or suspicious. All names given to the phenomenon described by the term 'incidental findings', and none without criticism. The neologism 'incidentaloma' describes something incidentally found through diagnostic imaging techniques, and has been used for over 30 years with increasing frequency, ${ }^{1}$ though not without controversy. ${ }^{2}$ Incidental findings can arise in any medical field - the medical professional or researcher simply has to discover something in the course of conducting a particular study that is beyond the aims of that study - though until recently they have occurred most frequently in radiology, as a byproduct of improved imaging capabilities. ${ }^{3}$ By analogy, new genetic sequencing technologies, which can also 'see' more than the particular aim of a particular study, are ripe for an explosion of incidental findings. ${ }^{4}$ We offer here a reflection on the difficulties posed by the various terms and definitions currently used for the phenomenon understood as an 'incidental finding'. The specific term that we endorse, particularly for use in genetics contexts, is 'secondary variants'. This term best avoids the problems raised by other options as well as accurately describing the phenomenon. The term 'incidental finding', though not our favorite term, is predominantly used below for convenience' sake.

Why is it so important to formulate standardized terminology for 'incidental findings'? The importance of the topic itself is indisputable, as evidenced by two recent symposiums, one on incidental findings in general, ${ }^{5}$ the other on the return of results and incidental findings from genetic and genomic research. ${ }^{6}$ The issue will not disappear in a hurry, if ever, precisely because of our increasing abilities in 'seeing, scanning, and sequencing. It is thus vital to agree on terminology. There is very little public guidance available at a governmental, professional or academic level, and what is available is inconsistent. ${ }^{7,8}$ Only standardized
${ }^{1}$ Centre for Biomedical Ethics and Law, Department of Public Health and Primary Care, Catholic University of Leuven, Leuven, Belgium; ²Department of Human Genetics, Catholic University of Leuven, Leuven, Belgium *Correspondence: Ms GM Christenhusz, Centre for Biomedical Ethics and Law, Department of Public Health and Primary Care, Catholic University of Leuven, Kapucijnenvoer 35, 3000 Leuven, Belgium. Tel: +32 16332919 ; Fax: +32 16 336952; E-mail: gabrielle.christenhusz@med.kuleuven.be

Received 19 December 2012; revised 4 March 2013; accepted 2 April 2013; published online 22 May 2013 terminology will allow international norms to be more easily compared and help in the drafting of research protocols and informed consent forms. ${ }^{8}$ Standardized terminology will also aid the comparison and further development of ideas coming from scientific articles, and clinical and research reports. ${ }^{9}$ Moreover, it is necessary to clarify current and future legal and ethical obligations, and to maintain the trust of participants and patients. ${ }^{10}$ Agreeing on appropriate terminology is thus no secondary issue in the incidental findings debate.

\section{JUST ANOTHER TYPE OF RESULT?}

The current debate about incidental findings is related to the ongoing debate about returning research results. The latter discussion has put forward various suggestions regarding the necessity to confirm analytic validity, clinical validity and utility, the right not to know, and the disclosure of research results that are clinically significant and actionable. ${ }^{11-13}$ This has informed the incidental findings debate, for example, that incidental findings that are clinically significant and actionable will be disclosed. However, there are two key differences between research results and incidental findings which argue that the two be considered separately: first, whether the finding falls inside or outside the domain or expertise of the researcher; second, whether there are clear or ambiguous follow-up obligations. ${ }^{13}$ We consider the differences to be significant reason enough not to use the word 'results' in a definition for 'incidental findings'. The use of the word 'results' could mask the 'incidental' or surreptitious nature of the discovery, inaccurately raising the expectations of what medical professionals or researchers might be expected to find in the course of a particular study.

\section{MORE THAN 'INCIDENTAL', 'UNANTICIPATED', OR 'UNEXPECTED'}

At the same time, there have been concerns raised with the descriptor 'incidental. ${ }^{14,15}$ This could be seen as minimizing the significance of the finding and is certainly less than appropriate in depicting certain potentially life-changing findings, such as the presence of a fatal disease or misattributed paternity. The use of the adjective 'incidental' is also complicated by those findings that are actually actively and intentionally sought for, while still not falling under the aim of the study. ${ }^{16}$ Such findings may be triggered by a chance remark by the patient or participant, or by a chance observation by the medical professional or 
researcher. But this chance event will then be actively and intentionally followed up. A term such as 'unsought for findings', promoted by the Health Council of the Netherlands and others, ${ }^{17}$ is thus also less than ideal. To find something you must first search for it, though this may begin subconsciously or intuitively. The prerequisite for making an 'unsought for finding' is that your eyes or mind be trained in a certain way so that you can recognize what it is you are looking at. ${ }^{18}$ Such training predisposes you to make findings within your domain of expertise. It is highly doubtful that a layperson would be able to identify an incidental finding on a radiological scan, and even more improbable that they be able to recognize the HIV genome from a blood sample, ${ }^{19}$ or somehow 'see' disease-causing variants in an exome sequence. ${ }^{16}$ It is similarly unlikely that a medical professional would discover an 'unsought for finding' outside of their domain of expertise. 'Incidental' findings are therefore not sheer 'co-incidences'.

There are likewise difficulties with labels such as 'unanticipated' or 'unexpected'. This is especially evident in clinical contexts. On the one hand, it could be reasonably expected of clinicians that nothing that is of potential clinical significance for their patients be 'unexpected'. ${ }^{13}$ The word 'unexpected' can thus cast doubt on the competency of medical professionals. It is also true that the frequency of some 'unanticipated findings' can actually be estimated based on their known frequency in the population, plus that some research and clinical activities are so liable to generating these types of findings that 'unanticipated' is then a misnomer. ${ }^{14}$ Moreover, sometimes medical tests are performed to try and isolate a diagnosis, when it is not just a matter of confirming a diagnosis but of genuinely trying to puzzle out what ails the patient. In such cases, everything is in a sense expected by the clinician, while patients may not be sure what to expect. Dividing findings in clinical contexts into something like 'unexpected' and 'expected' may act as a useful reminder to the doctor who returns the findings that some news will be truly unexpected to the patient (or parents), meaning that these findings should be returned in a different way to primary, 'expected' results. It can be a shock to learn unexpectedly, for instance, that one's 'healthy' child has a high risk of heart arrhythmia, ${ }^{20}$ or that a child with a neurodevelopmental disorder also has a deletion in the BRCA1 gene. ${ }^{21}$ In addition, maintaining a concept similar to unanticipated or unexpected findings in clinical contexts can aid in tempering the expectations of patients: to try and make it clear that while clinicians always strive for the advancement of their patients' health-related welfare, they can be expected to focus their attention on discrete clinical questions. 'No results' does not mean that every possible clinical condition and risk has been tested for and found negative. A term like 'off-target results' is a more promising suggestion than unanticipated or unexpected results. ${ }^{22}$ Incidental findings arise because of a field of vision (literally or figuratively) that is wider than the particular aim of the study or test. A word like 'off-target' is a reminder that vision is nonetheless targeted or focused, and what is most clearly seen is what the eyes target.

\section{RESPONDING TO THE EXPECTATIONS OF PATIENTS AND PARTICIPANTS}

The expectations of patients and participants should be borne in mind and responded to when devising names and definitions for 'incidental findings'. Research conducted with research participants shows that they generally expect researchers to disclose more than the latter expect to or are willing or able to. ${ }^{23-25}$ The therapeutic or diagnostic misconception, in which clinical research or screening is confused for clinical care, can be a problem in research and screening, a problem which can be compounded by discussing incidental findings with participants. Sometimes the therapeutic or diagnostic misconception occurs simply because being in an environment that reminds participants of medical environments can lead to clinical expectations, causing the participants to forget that they are in a research or screening environment. ${ }^{26}$ Some argue that merely mentioning the possibility of incidental findings to patients or participants will lead them to expect that incidental findings will be found, and that if no incidental findings are disclosed to them they will falsely assume that they are completely healthy. ${ }^{13,15,26-28}$

In fact, sometimes the expectations of the subjects of genetic tests may be so high that they have difficulty understanding the concept 'incidental finding', because they expect that everything significant will be found. This confusion is simply exacerbated when genetic tests are conducted without a clearly defined target in mind, but just to 'see if anything is wrong'. Examples include broad prenatal genetic screening and direct to consumer genetic testing bought by nominally 'healthy' people. In both cases, the recipients of the test results expect that anything 'wrong' will be reported to them, and that negative results can be interpreted as perfect health. These expectations are to a certain extent justified because the genetic test conducted is so broad. We maintain however that even in such cases it is helpful to communicate clearly that certain things are targeted by the genetic test and certain things are not. This will help temper the expectations of the recipients of genetic tests, and may also provide an opportunity to explain that a healthy lifestyle and family history can have as much of an impact on health as the right genes. $^{29}$

A further point to be considered in the context of the expectations of patients or participants is how narrow the definition of incidental findings should be, and whether this specificity can be communicated. An oft-quoted definition of incidental findings speaks of the 'potential health or reproductive importance' of what should be disclosed. ${ }^{13}$ Such findings clearly fall within the expertise of medical professionals. However, not all incidental findings are so clear-cut, nor are the expectations of patients or participants always so well defined. In cases where incidental findings are not so clear-cut, the question arises of whether medical professionals can restrict themselves to only dealing with the health or reproductive aspects of the incidental findings. An example is the incidental finding of misattributed gender caused by complete androgen insensitivity syndrome, which can raise deep-rooted identity issues alongside reproductive and health issues. A further complication is that public awareness of genetic risk factors is mixed, and public understanding is limited. ${ }^{30}$ This will impact what it is that patients or participants expect to have 'potential health or reproductive importance'. It is ultimately our view that for a definition of 'incidental findings' to be meaningful and useable for medical professionals and researchers, it should restrict itself as far as possible to that which has clinical and reproductive relevance. The broader or more confused expectations or concerns of patients and participants should be discussed in preparatory conversations on the return of results and incidental findings. Some room could be made for what the patients and participants themselves consider to be 'important.' ${ }^{\text {'1 }}$ However, if their high expectations are the sole determiner of the obligations of professionals, the latter will be overwhelmed in 'incidental findings.' ${ }^{32}$ Similarly, a modifier like 'unrelated' may be 
less helpful from a patient or participant point of view: an incidental finding will in some sense be 'related' to the original investigation and to the role of the medical professional involved.

\section{TIME FOR A NEW TERM: 'SECONDARY VARIANTS'}

What might be a meaningful term for the phenomenon described by 'incidental findings' in the context of new genetic sequencing techniques? We have thus far rejected the following terms: 'incidental', for potentially minimizing the weightiness of such a finding; 'incidental' and 'unsought for', because there is actually some degree of searching or purposeful looking involved; and 'unanticipated', 'unexpected', and 'unrelated', because of difficulties with what exactly can be expected of medical professionals and researchers, and what is expected by patients and participants.

The concept of 'result' was rejected when talking about 'incidental findings' in general. However, new genetic sequencing techniques call this stance into question. Their inherent open-endedness means that it can be difficult to distinguish 'incidental' from other findings. ${ }^{27}$ It can then be contended that nothing is 'incidental' because nothing falls outside the scope of the genetic test; everything is in some sense a 'result'. Filters can be imposed to try and limit the number of incidental findings, so that as much as possible only 'results' are discovered by new genetic sequencing techniques. We have argued elsewhere for the development of filters, though acknowledging that even filters will not make the phenomenon of incidental findings obsolete. ${ }^{33}$ Furthermore, the first objection to the term 'result' listed above, that results fall within the expertise of the professional while incidental findings may not, ${ }^{13}$ may soon no longer hold. In the coming age of global databases containing the combined interpreted data of many researchers, it would appear that researchers and clinicians will no longer be limited by their own individual expertise. However, there may still be unambiguous follow-up obligations. ${ }^{13}$ For instance, the disclosure of the high risk of a late-onset condition found unexpectedly in a child raises questions. ${ }^{21}$ Conversely, it will be clearly stated in the research or clinical protocol how results will be handled.

In contrast, ideas such as 'off-target' and 'clinical and/or reproductive importance' appear to be more promising. A further constructive addition to the debate is to stress that incidental findings are a sort of 'bonus'. They should not be counted upon, nor should their absence be interpreted as meaning anything specific. They are serendipitous in nature, resembling to some extent famous accidental discoveries of the past like Alexander Fleming's penicillin, Isaac Newton's gravity, or Achimedes' 'Eureka!' moment. 'Serendipitous' is indeed a fitting adjective; coined in the eighteenth century, it was intended to encompass not just the luck involved in such discoveries but also the astuteness needed to link together apparently innocuous or unrelated observations and facts to arrive at a valuable conclusion. ${ }^{18}$ Stressing the 'bonus' nature of incidental findings will furthermore aid in dispelling the notion that they are somehow 'risks' of medical procedures or 'caused' by medical procedures, as is sometimes suggested. ${ }^{14,24,34}$ They also need not be 'abnormalities;' the precise nature of some incidental findings, and whether they are good news or bad, will be initially unclear. Incidental findings refer to conditions and risks that existed before the medical procedure was carried out: the medical procedure simply brings them to light. This can help to put professional obligations surrounding incidental findings into perspective: the disclosure of an incidental finding is more a 'bonus' than an attempt to set something right that the medical professional is guilty of causing.

We ultimately endorse the term 'secondary variants'. Johnston et al. ${ }^{35}$ use this term in a recent article on their attempt to develop approaches for the analysis and return of whole-exome sequencing results. They make a distinction between 'the mutation causing the disorder for which the sequencing was performed', the so-called 'primary variant', and 'other clinically important results', referred to as 'secondary or so-called incidental variants'. A link with 'primary variants', the aim of the particular genetic test, is maintained, unlike with a term like 'unsought for', and without the use of potentially confusing words like 'results' and '(un)related.' 'Secondary variants' is thus an apt term in a field in which it is becoming increasingly ambiguous what is and is not a research 'result. It is certainly a suitable term for use in clinical contexts, in which a similar ambiguity revolves around what precisely is and is not a 'result' from the point of view of the clinician. In addition, the word 'variants' is sufficiently neutral to avoid the possible difficulties of 'risk' and 'abnormality', while still suggesting that something important has been found, unlike the connotations of 'incidental'. It remains to be seen how widespread this term might become.

\section{CONFLICT OF INTEREST}

The authors declare no conflict of interest.

\section{ACKNOWLEDGEMENTS}

This work was supported by FWO Flanders, project number G029107 and G.0594.09. K Devriendt is senior clinical investigator of the FWO-Vlaanderen and of the K.O.O.R U.Z.Leuven. We thank the anonymous reviewers for their valuable comments and suggestions.

1 Aron DC: Preface. Best Prac Res Clin Endocrinol Metabol 2012; 26: 1-2.

2 Mirilas P, Skandalakis JE: Benign anatomical mistakes: incidentaloma. Am Surg 2002; 68: 1026.

3 Stone $\mathrm{JH}$ : Incidentalomas - Clinical correlation and translational science required. N Engl J Med 2006; 354: 2748-2749.

4 Kohane IS, Hsing M, Kong SW: Taxonomizing, sizing, and overcoming the incidentalome. Genet Med 2012; 14: 399-404.

5 Wolf S: Introduction: the challenge of incidental findings. J Law Med Ethics 2008; 36: 216-218.

6 Wolf SM: The past, present, and future of the debate over return of research results and incidental findings. Genet Med 2012; 14: 355-357.

7 Lawrenz F, Sobotka S: Empirical analysis of current approaches to incidental findings. J Law Med Ethics 2008; 36: 249-255.

8 Zawati $\mathrm{MH}$, Knoppers BM: International normative perspectives on the return of individual research results and incidental findings in genomic biobanks. Genet Med 2012; 14: 484-489.

9 Christenhusz GM, Devriendt K, Dierickx K: To tell or not to tell? A systematic review of ethical reflections on incidental findings arising in genetics contexts. Eur J Hum Genet 2013; 21: 248-255.

10 Knoppers BM, Dam A: Return of results: towards a lexicon? J Law Med Ethics 2011; 39: 577-582.

11 Knoppers B, Joly Y, Simard J, Durocher F: The emergence of an ethical duty to disclose genetic research results: international perspectives. Eur J Hum Genet 2006; 14: 1170-1178.

12 Ravitsky V, Wilfond B: Disclosing individual genetic results to research participants. AJOB 2006; 6: 8-17.

13 Wolf S, Lawrenz F, Nelson C et al: Managing incidental findings in human subjects research: analysis and recommendations. J Law Med Ethics 2008; 36: 219-248.

14 Parker L: The future of incidental findings: should they be viewed as benefits? J Law Med Ethics 2008; 36: 341-351.

15 Sharp H, Orr R: When 'minimal risk' research yields clinically-significant data, maybe the risks aren't so minimal. AJOB 2004; 4: W32-W36.

16 Lyon GJ: There is nothing 'incidental' about unrelated findings. Pers Med 2012; 9: 163-166.

17 Health Council of the Netherlands: The 'ThousandDollar Genome': an Ethical Exploration. The Hague: Centre for Ethics and Health, 2010.

18 Stoskopf MK: Observation and cogitation: how serendipity provides the building blocks of scientific discovery. ILAR J 2005; 46: 332-337.

19 Kolata G: Genes now tell doctors secrets they can't utter. The New York Times, 2012.

20 Bush LW, Rothenberg KH: Dialogues, dilemmas, and disclosures: genomic research and incidental findings. Genet Med 2012; 14: 293-295.

21 Bruno DL, Stark Z, Amor DJ et al: Extending the scope of diagnostic chromosome analysis: detection of single gene defects using high-resolution SNP microarrays. Hum Mutat 2011; 32: 1500-1506.

22 Mayer AN, Dimmock DP, Arca MJ et al: A timely arrival for genomic medicine. Genet Med 2011; 13: 195-196. 
23 Bovenberg J, Meulenkamp T, Smets E, Gevers S: Biobank research: reporting results to individual participants. Eur J Health Law 2009; 16: 229-247.

24 Kirschen M, Jaworska A, Illes J: Subjects' expectations in neuroimaging research. J Magn Reson Imaging 2006; 23: 205-209.

25 Matsui K, Lie RK, Kita Y, Ueshima H: Ethics of future disclosure of individual risk information in a genetic cohort study: a survey of donor preferences. J Epidemiol 2008; 18: 217-224.

26 Shaw R, Senior C, Peel E et al: Ethical issues in neuroimaging health research: an IPA study with research participants. J Health Psychol 2008; 13: 1051-1059.

27 Cho MK: Understanding incidental findings in the context of genetics and genomics. J Law Med Ethics 2008; 36: 280-285.
28 Miller FG, Mello MM, Joffe S: Incidental findings in human subjects research: what do investigators owe research participants? J Law Med Ethics 2008; 36: 271-279.

29 Becker F, van El CG, Ibarreta D et al: Genetic testing and common disorders in a public health framework: how to assess relevance and possibilities. background document to the ESHG recommendations on genetic testing and common disorders. Eur J Hum Genet 2011; 19: S6-44.

30 Smerecnik CM, Mesters I, de Vries NK, de Vries $\mathrm{H}$ Educating the general public about multifactorial genetic disease: applying a theory-based framework to understand current public knowledge. Genet Med 2008; 10: 251-258.

31 Lavieri R, Garner S: Ethical considerations in the communication of unexpected information with clinical implications. AJOB 2006; 6: 46-48.
32 Sonnenberg A: Personal view: 'don't ask, don't tell'-the undesirable consequences of incidental test results in gastroenterology. Aliment Pharmacol Ther 2004; 20: 381-387.

33 Christenhusz GM, Devriendt K, Vermeesch J, Dierickx $\mathrm{K}$ : Why genomics shouldn't get too personal: In favor of filters: Re: invited comment by Holly K. Tabor et al. in American Journal of Medical Genetics Part A Volume 155. Am J Med Genet A 2012; 158A: 2641-2642.

34 Meacham M, Starks H, Burke W, Edwards K: Researcher perspectives on disclosure of incidental findings in genetic research. J Empir Res Hum Res Ethics 2010; 5: 31-41.

35 Johnston JJ, Rubinstein WS, Facio FM et al: Secondary variants in individuals undergoing exome sequencing: screening of 572 individuals identifies high-penetrance mutations in cancer-susceptibility genes. Am J Hum Genet 2012; 91: 97-108. 